\title{
Tangence
}

\section{La notion d'écrivain chez les élèves de cinq à neuf ans1}

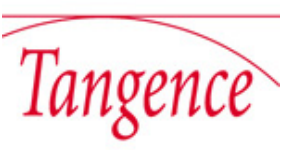

\section{Flore Gervais}

Numéro 67, automne 2001

L'écriture pour la jeunesse : de la production à la réception

URI : https://id.erudit.org/iderudit/009615ar

DOI : https://doi.org/10.7202/009615ar

Aller au sommaire du numéro

Éditeur(s)

Presses de l'Université du Québec

ISSN

0226-9554 (imprimé)

1710-0305 (numérique)

Découvrir la revue

Citer cet article

Gervais, F. (2001). La notion d'écrivain chez les élèves de cinq à neuf ans1.

Tangence, (67), 54-68. https://doi.org/10.7202/009615ar
Résumé de l'article

Résumé

Cet article expose les résultats d'une recherche sur la représentation mentale de l'écrivain pour la jeunesse chez 206 élèves âgés de cinq à neuf ans d'une école métropolitaine de milieu socio-économique moyen. Ces derniers ont été invités à répondre oralement à quatre questions. 1) Qu'est-ce qu'un écrivain ? 2) La personne qui écrit pour les jeunes doit-elle être jeune elle-même? Pourquoi ? 3) Où l'écrivain trouve-t-il ses idées? 4) Quelle(s) diffêrence(s) y a-t-il entre un livre pour enfants et un livre pour adultes ? Une analyse de fréquence des réponses à chacune de ces questions met en lumière des constances qui sont révélatrices d'une vision à la fois naïve, originale et souvent juste de l'écrivain et de l'écrit. Nous illustrons les principaux résultats de cette enquête à l'aide des mots des enfants. 


\section{La notion d'écrivain chez les élèves de cinq à neuf ans ${ }^{1}$}

\section{Flore Gervais, Université de Montréal}

Bien avant d'entrer en maternelle, les enfants sont mis en contact avec plusieurs formes de textes ${ }^{2}$. En effet, il suffit d'assister au rituel de la lecture avant le coucher du petit, ou encore à l'heure du conte de la bibliothèque ou de la garderie du quartier, pour s'apercevoir que beaucoup d'enfants sont familiers avec la narration orale d'histoires et, notamment, la lecture orale par l'adulte de récits de toutes sortes. Par conséquent, nous pouvons penser que, dès leur entrée à l'école, les enfants possèdent déjà une certaine représentation mentale de l'écrit et de l'écrivain. Mais laquelle?

En outre, s'il est vrai que les représentations mentales des jeunes se complexifient et que, jusqu'à un certain âge, leur champ s'élargit au fil des années ${ }^{3}$, il nous a semblé intéressant de voir comment évoluaient, selon leur cycle scolaire, les représentations mentales que les jeunes écoliers se faisaient des auteurs et de leurs livres. Par ailleurs, comme la recherche a très souvent montré que les filles et les garçons affichaient des habitudes différentes de lecture ${ }^{4}$, il nous a également paru opportun d'analyser leur conception respective de l'écrivain.

Pour ce faire, nous avons orienté notre étude de manière à embrasser trois grands objectifs: a) mettre au jour le réseau de connaissances sur l'écrivain et sur l'écrit partagées par des groupes d'élèves d'un âge déterminé; b) observer dans quelle mesure

1. L'auteure tient à remercier pour leur précieuse collaboration à cette recherche les élèves de l'école Laurier à Montréal, d'une part, Isabelle Darveau, son assistante de recherche, d'autre part.

2. Voir Émilia Ferreiro, "Literacy development: Psychogenesis ", dans Yetta Goodman, How Children Construct Literacy, Newark (Del.), International Reading Association, 1990, et Guy Boudreau et al,, Réussir dès l'entrée clans l'écrit, Sherbrooke, Éditions du CRP, Université de Sherbrooke, 1993.

3. Voir Marcel Thouin, La didactique des sciences de la nature au primaire, Québec, Éditions Multimonde, 1997.

4. Flore Gervais, École et habitudes de lecture. Etude sur les perceptions diélèves québécois de 9 à 12 ans, Montréal, Chenelière/McGraw-Hill, 1997. 
leurs représentations de ces deux réalités évoluent entre l'âge de cinq et neuf ans; c) voir si leurs représentations diffèrent selon le sexe des répondants. Dans les pages qui vont suivre, nous exposerons d'abord notre méthode de collecte et de traitement des données, puis nous présenterons les résultats obtenus en même temps que nous les commenterons à la lumière des théories du développement de l'enfant et de celles du traitement de l'information ${ }^{5}$.

\section{Méthode}

\section{a) Collecte des données}

La population étudiée provient d'une école montréalaise fréquentée par des enfants issus d'un milieu socio-économique moyen ". La recherche s'est effectuée auprès de 206 élèves, dont 100 garçons et 106 filles inscrits à l'éducation préscolaire et au primaire. Ces derniers se répartissaient comme suit: 70 élèves de maternelle (5-6 ans), 130 du primaire dont 94 élèves de premier cycle ( $7-8$ ans) et 42 de second cycle $(8-9$ ans). La collecte des données s'est effectuée à l'aide d'un questionnaire qui ne comportait que quatre questions. Compte tenu de la durée limitée de l'attention que de jeunes élèves sont en mesure d'accorder à un questionnement de type cognitif, il ne fallait pas rallonger indûment le temps de réponse. Ces quatre questions recoupaient certains aspects de la notion d'écrivain et d'écrit. Elles étaient libellées de la façon suivante: 1) Qu'est-ce qu'un écrivain? 2) La personne qui écrit pour les jeunes doit-elle être jeune elle-même? Pourquoi? 3) Où l'écrivain trouve-t-il ses idées? 4) Quelle(s) différence(s) y a-t-il entre un livre pour enfants et un livre pour adultes?

Les questions étaient posées oralement pour deux raisons: d'une part, le jeune âge des répondants, qui explique le manque de maîtrise du geste graphique; d'autre part, le type d'informations que nous cherchions à mettre en relief. En outre, nous avons choisi d'effectuer notre collecte de données à partir d'un questionnement collectif par groupe-classe. Ce dernier permet

5. Pour une synthèse, voir Louis Dubé, Psychologie de l'apprentissage de 1880 à 1980, Sillery, Presses de l'Université du Québec, 1986, p. 290-313.

6. Voir Conseil scolaire de l'île de Montréal, Classification des écoles primaires selon l'ordre décroissant de l'indice de défavorisation - Inscription au 30 septembre, 1992-1993, 1993. 


\section{Tangence}

d'obtenir des données non seulement sur ce que certains élèves connaissent d'une réalité, mais aussi sur la capacité d'un groupe à se construire un réseau de connaissances sur cette réalité à l'aide des réponses des autres. Ce mode d'enquête offre deux autres avantages: d'une part, il permet d'élargir les connaissances du groupe par enseignement réciproque ${ }^{7}$ grâce à la discussion en commun $^{8}$; d'autre part, il permet de saisir une image "impressionniste" ou "globale" des représentations mentales que se font sur l'écrivain non pas des individus pris isolément, mais des groupes d'élèves d'un âge déterminé.

La rigueur de la démarche assure, en clépit de certaines limites, la validité des résultats. L'enquête s'est effectuée dans le cadre d'un projet d'école sur la littérature de jeunesse. Les questions ont été posées, dans le même ordre, à tous les groupes, par nous-même; nous avons recueilli toutes nos données dans le courant d'un même mois, auprès de groupes différents d'élèves dont lâge correspondait à ceux que nous avions ciblés. La collecte des données s'est effectuée selon un mode transversal et non pas longitudinal. Dans toutes les classes, nous avons été reçue avec enthousiasme et les réponses des élèves ont été enregistrées sur magnétophone, puis transcrites à l'aide d'un logiciel de traitement cle texte.

\section{b) Traitement des données}

Une fois les corpus sonores transcrits, nous avons suivi, d'une part, les étapes caractéristiques de l'analyse de contenu: lecture flottante", élaboration et validation d'une grille d'analyse sur un extrait du corpus, analyse, codification et catégorisation des énoncés en termes de réponses et de sous-réponses, calcul des occurrences selon leur fréquence d'apparition, et selon une moyenne virtuelle par enfant. Aucune corrélation statistique n'a été effectuée sur les données. Comme l'objet d'analyse était cons-

7. Sylvie Blain, L'impact de la rétroaction verbale des pairs sur l'amélioration des compositions des élèves de cinquième année en immersion française, thèse de doctorat, Université de Montréal, 1997, p. 244.

8. Sur ce concept, voir Hans Aebli, Didactique psycbologique, Neuchâtel, Delachaux et Niestlé, 1966, p. 93.

9. Laurence Bardin, Lanalyse de contenu, Paris, Presses universitaires de France, 1977. 
titué d'énoncés qui comportaient plus d'un élément conceptuel, nous avons élaboré et mis en place, d'autre part, pour chacune des quatre questions, un code sémantico-syntaxique susceptible de rendre compte de façon pertinente et efficace non seulement des réponses, mais de tous les types de sous-réponses.

Voici les résultats qualitatifs que nous avons obtenus après une analyse de fréquence des types de réponses répertoriés. Comme les groupes ne contiennent pas le même nombre d'individus, il nous a fallu, pour rendre comparables nos données, les uniformiser à l'aide d'une moyenne (virtuelle) de réponses et de sous-réponses par élève.

\section{Résultats généraux}

Une analyse comparative globale des résultats tend à montrer que le nombre des réponses données pour l'ensemble des quatre questions croît par ordre et cycle d'enseignement, d'une moyenne virtuelle de $1,21,1,27$ et 1,81 par élève. De plus, les filles ont tendance à répondre un peu plus souvent que les garçons: en moyenne 1,44 fois côté fille, 1,27 fois côté garçon. Si le sexe des répondants semble avoir peu joué sur les réponses prises globalement, le type de sous-questions posées semble, par contre, avoir eu un certain impact sur la motivation des élèves à approfondir davantage leurs réponses à l'une ou à l'autre des questions. Les différences s'observent surtout entre les ordres et cycles d'enseignement.

Un calcul du nombre de sous-réponses par sous-questions tend à montrer que des élèves ont été plus sensibles à certaines questions qu'à d'autres. Ainsi, la question 1: "Qu'est-ce qu'un écrivain?" a stimulé davantage de sous-réponses chez les élèves du second cycle. Comment expliquer cette différence? On peut penser qu'à leur âge, les élèves ont été plus d'une fois soumis à des exercices de définition. Cette opération mentale n'exige-t-elle pas une plus grande expérience d'observation du monde pour en reconnaître les règles générales, d'une part, en extraire les particularités, d'autre part? Ajoutons que les élèves de cette école avaient déjà, comme ceux de bien d'autres écoles, eu l'occasion de recevoir la visite d'une auteure.

La question 2: "La personne qui écrit des livres pour des jeunes doit-elle être jeune elle-même?" a suscité, en moyenne, 
pratiquement le même nombre de sous-réponses de la part de tous les groupes interrogés. Ce fat est probablement dû à la teneur même de la question. En effet, cette question n'exigeait, à toute fin pratique, qu'une réponse par "oui "ou par "non". Cependant, grâce à nos sous-questions, les élèves ont quand même commenté leur réponse. Nous verrons plus loin comment une question fermée, en apparence sans grand potentiel de réponses variées, peut, grâce à des sous-questions incitatives, s'avérer une précieuse source d'information non seulement sur les représentations mentales des élèvés, mais aussi sur leur capacité d'expression.

Par ailleurs, les questions 3: "Où trouvent-ils leurs idées?" et, plus particulièrement, 4: "Quelles différences y a-t-il entre un livre pour enfants et un livre pour adultes?" sont celles qui ont le plus inspiré les répondants. De plus, elles ont suscité beaucoup plus la participation des élèves du premier cycle. Comment expliquer ce phénomène? Serait-ce à cause d'un effet d'entraînement? Nous sommes portée à répondre non car, comme nous le verrons, la quatrième question, qui aurait dû inspirer plus de réponses, en a moins suscitées. Dès lors, serait-ce plutôt parce que les questions 3 et 4 , plus concrètes, étaient plus intéressantes? Si oui, pourquoi le seraient-elles davantage pour les élèves de premier cycle? Enfin, se pourrait-il que ces débutants en écriture et en lecture, tout imprégnés qu'ils sont des joies et des tribulations reliées au processus d'écriture et au décodage en lecture, soient davantage capables d'en parler?

Nous verrons, dans ce qui suit, comment une analyse plus fine des résultats pourra confirmer ces hypothèses ou appuyer l'interprétation que nous en faisons, d'une part, apporter des connaissances nouvelles sur les représentations mentales que les jeunes écoliers se font de l'écrivain et de l'écrit, d'autre part.

\section{Résultats par question Première question : "Qu'est-ce qu'un écrivain ? "}

Les élèves ont répondu majoritairement en citant 26 objets d'écriture ou genres littéraires qui caractérisent son travail. Les cinq catégories d'écrits les plus souvent mentionnées (les livres, les poèmes, les histoires, les dictionnaires, le théâtre) ne l'ont été qu'entre deux et cinq fois, et les 21 autres genres de productions ne l'ont été qu'une fois chacun. Ce large éventail de catégories lit- 
téraires ou d'autres objets d'écriture évoqués par les jeunes élèves montre qu'ils ont été mis en contact avec plusieurs types d'écrit dès la maternelie. En effet, ceux de maternelle évoquent presque autant de catégories littéraires ou d'autres formes de productions écrites que les élèves de second cycle du primaire (16 contre 17); par contre, ils font référence - sauf exception pour le poème, la bédé et l'alphabet - surtout aux livres, aux textes, aux histoires en général, tandis que les élèves du second cycle manifestent leur connaissance d'une plus grande variété d'ouvrages particuliers comme les romans, les dictionnaires, les encyclopédies, les publicités, les sites Internet, les cédéroms; ils iront jusqu'à évoquer les bulles de bédé.

En outre, d'autres élèves définissent l'écrivain en fonction de son ou de ses publics cibles, c'est-à-dire qu'ils reconnaissent que certains écrivains "écrivent pour les jeunes", alors que d'autres s'adressent aux "adultes".

Enfin, une analyse plus fine montre combien les élèves, tout jeunes encore, sont capables de représentations mentales complexes. En effet, ils se montrent aptes à porter un jugement de valeur sur les écrits: "C'est quelqu'un qui fait des livres très bien" ou encore "qui écrit des très beaux poèmes". Ils parviennent également à reconnaître certaines étapes du processus d'écriture: "Il pense à ce qu'il va écrire; après ça, il le met en pages; après ça, il dit: "Ah non! C'est pas bon". Il repense, il le tape. Si ça a du sens, il va le publier; il va demander à l'éditeur si c'est bon comme livre". Ils sont également capables de nuancer leurs propos. Deux exemples: "Il vole pas réellement", rétorque un élève à un autre pour qui l'écrivain est un voleur qui capte les caractéristiques des passants pour les réutiliser dans son livre; "il y a des écrivains qui écrivent dans La courte échelle", ajoute un élève de maternelle. Ainsi, dans le premier exemple, les termes pas réellement viennent nuancer laffirmation précédente, tandis que dans le second exemple, le déterminant partitif des pourrait signifier que l'écolier sait qu'il existe plus d'une maison d'édition.

\section{Deuxième question : "La personne qui écrit des livres pour des jeunes doit-elle être jeune elle-même? "}

Plus les élèves sont jeunes, plus ils répondent en fonction de leur propre capacité à écrire, voire à lire. Quand ils estiment que ce doit être un adulte qui écrit pour les jeunes, ils expliquent 
ainsi leur point de vue: "parce que les enfants, i sont pas trop bons pour écrire" ou "parce que les [enfants de] maternelle, i savent pas écrire». On entendra même une enfant de maternelle dire "i faut que ce soit des adultes parce que les jeunes i peuvent faire des fautes ". Cette dernière assertion pourrait laisser entendre que, même en maternelle, certains enfants comprennent qu'écrire veut dire écrire non seulement selon une convention, mais aussi écrire sans fautes! Par ailleurs, d'autres tiendront le discours inverse, mais s'appuieront quand même sur leur propre expérience: la personne qui écrit des livres pour des jeunes peut être jeune elle-même, parce que "Moi, j'en ai déjà écrit cinq (des histoires)"; et puis, "On peut écrire des lettres majuscules aussi, puis des lettres carrées". Enfin certains élèves de maternelle, tout à la joie de nous dire qu'ils ont appris à lire, semblent oublier que la question portait sur l'écrivain : "Nous aussi on a appris à lire. On a appris le $\mathrm{A}$ ! Comme cette réponse n'apporte aucune information quant à l'âge que doit avoir l'écrivain pour les jeunes, nous l'avons classée, comme certaines autres, dans les "ambiguës". Elle révèle le stade de développement que Piaget nomme "le syncrétisme du raisonnement", qui amène l'enfant à répondre par "analogie immédiate ${ }^{10}$ *. En effet, le jeune enfant lie ici l'âge de l'écrivain qui peut écrire à sa propre habileté à lire ce que ce dernier écrit.

À l'inverse, les élèves plus âgés manifestent à travers leurs réponses leur aptitude à nuancer leur pensée. Voici quelques exemples qui illustrent cette observation. L'un dira: "Ça peut être un adulte comme un adolescent "; un deuxième explique: "C'est pas nécessaire d'être jeune, mais il faut se tenir au courant des actualités et se rappeler de ce qu'on a fait, parce que si t'es au courant d'actualités puis que t'as 70 ans, c'est pas grave, parce que tu te rappelles de ce qui intéresse les jeunes". Un troisième élève ira jusqu'à appuyer son argumentation d'une condition: "Pas obligé d'être jeune ou vieux, mais quand même garder un esprit d'enfant "; un dernier ajoutera: "Quand on est grand, on a plus d'idées dans la tête». Ici, nous observons que l'élève va plus loin que la simple convention en vertu de laquelle il forme ses lettres ou écrit sans fautes, comme le faisait remarquer l'élève de maternelle: il est capable d'appréhender la phase initiale du processus d'écriture caractéristique du travail de l'écrivain.

10. Jean Piaget, Le langage et la pensée chez l'enfant, Neuchâtel (Suisse), Delachaux et Niestlé, 1970, p. 141. 
D'autres réponses à cette deuxième question témoignent, d'une part, des connaissances littéraires que peuvent avoir les élèves: "[...] Il y a des jeunes comme Anne Frank qui peuvent faire des livres n. Elles nous renseignent, d'autre part, sur leur sens inouï de la métaphore. Il y a l'élève qui, comme on l'a vu, compare l'écrivain à un voleur, mais il y a aussi celle qui renchérit sur l'idée d'un de ses compagnons, en ajoutant: "Non. Comme ma grand-maman, elle, elle fait des robes comme si les robes étaient des histoires, puis elle les vend". Cet élève associe le métier d'écrivain non plus à la figure du voleur, mais à celle d'une couturière. L'écrivain peut être jeune ou vieux, peu importe pour cette élève: le métier d'écrivain a le même statut que celui de couturière, puisqu'il s'agit d'un commerce dont la valeur d'échange est fondée sur l'argent.

\section{Troisième question : "Où l'écrivain trouve-t-il ses idées ?"}

À elle seule, cette question a provoqué deux fois plus de sous-réponses que les trois autres réunies. Après avoir répondu, dans un premier temps, de façon pragmatique, voire tautologique, les élèves se rendent compte que l'écrivain peut trouver ses idées ailleurs que "dans sa tête", "dans son cerveau", "dans ses pensées" ou "dans ses idées". Ils mentionnent alors des objets, des lieux, des événements, des personnes ou leurs caractéristiques, y compris les propres qualités de l'écrivain. Enfin, ils pensent que celui-ci peut aussi poser des gestes plus systématiques ou introspectifs.

Les élèves de maternelle évoquent surtout des objets que l'écrivain peut regarder et qu'ils ont eux-mêmes sous les yeux: "des dessins", "un tableau" (qui est en l'occurrence rempli de leurs dessins), "des vieilles choses d'enfants" (qui se trouvent dans un coffre à jouet dans un coin de la classe), et ils ajoutent "une ville", "des cadres", "des masques", "une pierre", etc. Il est intéressant de noter qu'ils puisent dans leur environnement immédiat les réponses à cette question, comme s'ils étaient des écrivains à l'œuvre au moment même où la question leur est adressée.

Les élèves du premier cycle énumèrent eux aussi des objets. Cependant, quoique encore reliés à leur expérience personnelle, 
certains de ces objets tombent moins immédiatement sous le regard des sujets interrogés; ils sont mentionnés en plus grand nombre et révèlent une plus grande variété de sources. Les élèves disent que les écrivains puisent leurs idées dans leurs lectures et, plus particulièrement, dans des types d'ouvrages comme "les livres", "les dictionnaires", "les magazines", puis dans des types de récits ou dans leurs composantes comme "les histoires" ou "les personnages". Ils croient aussi que l'écrivain peut s'inspirer de phénomènes culturels ou médiatiques comme "le cinéma", "les spectacles", "les pièces de théâtre", "la télévision" et "la radio. Par ailleurs, à l'instar des élèves plus jeunes, ils ont tendance à évoquer des lieux qu'ils connaissent en se référant à des affiches ou des couvertures de livres qui leur tombent sous les yeux immédiatement après que la question leur ait été posée. Ils disent que l'écrivain peut puiser ses idées "dans la forêt", "sur les feuilles des arbres", "dans la nature ", "autour de lui", "au maga$\sin$ ", etc. Bref, ces réponses tendent à montrer qu'un réseau conceptuel est difficilement détachable, du moins à cet âge, non seulement de ce que vivent les élèves, mais de ce qu'ils vivent bic et nunc. En effet, on observe très peu de distance entre ce qu'ils disent et ce qu'ils vivent dans l'immédiat.

Ce même phénomène se retrouve chez les élèves du second cycle, mais à un degré moindre, car leurs représentations mentales s'approfondissent, s'élargissent et se complexifient. On sent poindre une plus grande capacité à se détacher de soi pour se mettre véritablement dans la "peau" de l'écrivain. Cette nouvelle empathie pour la personne de l'écrivain se retrouve à travers des mentions de genres de récits et de lieux, certes, mais aussi à travers des évocations d'événements, de personnes-ressources et de leurs caractéristiques physiques ou psychologiques, ou encore à travers des suggestions d'activités plus complexes. Quant aux genres de récits, sources probables d'inspiration de l'écrivain, les élèves se montrent capables de raffiner leurs réponses jusqu'à différencier "le conte" de "la légende"; pour ce qui est des lieux, l'écrivain peut aussi sortir de la classe pour chercher ses idées: à leur avis, il peut les trouver "n'importe où " comme "dehors", "sur la terrasse». Ce qui frappe surtout, c'est que ce n'est qu'à cet âge qu'ils semblent reconnaître que l'écrivain peut s'inspirer surtout d'événements: leurs suggestions vont de la simple "bataille" jusqu'aux "choses extraordinaires qui se passent", ou encore évoquent "ce qu'il trouve beau "dans sa vie quotidienne" ou dans sa 
vie "courante", notamment "les fêtes de l'année" comme "la Saint-Valentin et Noël. Outre les événements, certains élèves du second cycle ont parlé du recours "à son entourage" qu'il peut interroger ou mettre en scène. De plus, les élèves ont mentionné qu'il peut entreprendre, pour étoffer ses personnages, une activité plus complexe, comme une enquête "dans une classe de chaque école". Enfin, un élève va jusqu'à dire que l'écrivain n'a finalement qu'à faire de l'introspection et à s'inspirer de "ses propres qualités".

\section{Quatrième question : “Quelle(s) différence(s) y a-t-il entre un livre pour enfants et un livre pour adultes ? "}

À mesure qu'ils grandissent, les élèves semblent légèrement plus motivés et habiles à décrire les différences qu'ils observent entre ces deux types de livres; en effet, la moyenne de leurs sous-réponses crồt de 0,5 , à 0,6 et à 0,7 par élève et par ordre d'enseignement. Mais quels sont les paramètres qui distinguent, à leurs yeux, un livre pour enfant d'un livre pour adulte? Les élèves sont-ils plus sensibles à certains de ces paramètres selon leur âge?

Indépendamment de l'ordre d'enseignement, nous pouvons regrouper les commentaires des élèves sous quatre aspects: les caractéristiques formelles, le contenu des livres, les autres supports de récits et les expériences personnelles.

\section{Caractéristiques formelles}

Les élèves se représentent le livre pour enfants rempli d'images et de couleurs. Leurs commentaires, qui portent sur ces deux critères, présentent le plus grand nombre d'occurrences dans les réponses à cette quatrième question. Ainsi un élève de maternelle affirme avec beaucoup de conviction que le livre pour adultes comporte de "l'écriture" alors que le livre pour enfants, "des dessins". D'autres tentent aussitôt de nuancer son assertion en reconnaissant que le livre pour adultes peut contenir aussi des images; ils ajoutent: "[mais] pour les adultes, i sont pas en couleurs", "les livres pour adultes, c'est noir et blanc".

Outre la présence de l'image et de la couleur, les aspects extérieurs du livre comme son volume, c'est-à-dire l'espace qu'il 
occupe, sa dimension, son épaisseur, et même l'épaisseur de ses pages, constituent des éléments qui, selon nos répondants, varient en fonction du lecteur cible. Ainsi, ces derniers reconnaissent que, si le livre pour adultes est plus épais ("Pour les petits enfants, parfois c'est mince; puis pour les grands, c'est épais"), ses pages sont minces: "Pour les enfants, les pages sont un peu plus épaisses puis, pour les adultes, i sont minces, minces. Le matériau et la plus ou moins grande flexibilité de sa couverture sont aussi reconnus par certains élèves comme caractéristiques du livre pour enfants: "Les livres pour enfants, c'est comme du carton, puis pour les adultes, c'est plus souple". Ajoutons à cela la longueur: les plus âgés reconnaissent que le "nombre de pages" et "la longueur des textes" font la différence entre livres pour enfants et livres pour adultes.

\section{Le contenu du livre}

Sur le plan du contenu, les élèves mentionnent des phénomènes très variés. Ils relèvent des différences qui vont des fonctions de l'écrit, des thématiques et des genres littéraires aux aspects linguistiques, c'est-à-dire graphiques, lexicaux et structuraux.

Pour ce qui est des fonctions de l'écrit, ils reconnaissent aux livres pour enfants la fonction narrative fictionnelle qu'ils opposent à la fonction explicative ou informative des livres pour adultes. Ils affirment: "Dans les livres pour les petits, c'est des histoires", "[...] c'est plus imaginaire", alors que "dans les livres pour adultes, ça explique des choses", "[...] c'est plus vrai". Ils attribuent aussi la fonction ludique aux livres pour enfants: "Les livres pour adultes, c'est beaucoup plus sérieux, pis les livres pour les enfants, c'est plus rigolo", "c'est plus drôle ". Ils opposent aussi cette intention de faire rire, qu'ils croient propre aux livres pour enfants, à celle de faire peur, qu'ils attribuent le plus souvent aux livres pour adultes.

Quant aux thématiques, la violence, la guerre et l'amour s'avèrent, selon eux, les trois sujets caractéristiques des livres pour adultes: "Les livres pour enfants sont pas violents, y a aucune guerre", dira l'un; "Il y a plus d'amour dans des livres pour adultes", ajoutera un autre. De plus, les élèves attribuent aux livres pour adultes une complexité des thématiques supérieure à celle des livres pour enfants. Un élève déclare: “Les 
sujets sont plus complexes chez les adultes"; l'autre ajoute: "Moi, je trouve que les livres pour adultes, c'est plus compliqué à comprendre des fois; les livres pour enfants, c'est comme plus adapté». Cette dernière affirmation tend à montrer que le jeune croit que l'écrivain pour la jeunesse se soucie de prendre en compte ses intérêts, voire sa capacité de lecture. Est-ce le cas? Même si le phénomène Harry Potter montre qu'un même livre peut plaire indistinctement aux enfants et aux adultes ", la question demeure posée puisque l'adaptation dite "intentionnelle " n'a jamais fait l'unanimité des écrivains.

Pour illustrer les différences, certains élèves font spontanément référence aux genres de récits, notamment au roman policier et au conte. Cependant, ils ne partagent pas tous la même opinion ou présentent des opinions plus nuancées sur la constitution de certains genres. Ainsi un élève reconnaît que le roman policier est destiné aux adultes, parce qu'il est porteur de violence: "Y a plus de violence dans des livres pour adultes comme dans les romans policiers", alors qu'un autre nuance: "[...] Y a des romans policiers qui sont pour nous encore ". Par ailleurs, d'autres constatent que le conte peut ne pas contenir d'images, même s'il s'adresse aux enfants: "Y a des contes pour enfants pas d'images". Quoi qu'il en soit, malgré cette divergence de point de vue sur la question, les réponses prouvent la compétence des élèves à reconnaître certaines caractéristiques du conte et du roman policier.

Par ailleurs, les réponses indiquent que les élèves sont sensibles à des différences qui portent sur les aspects plus linguistiques des écrits, comme les signes graphiques et les indices lexicaux. Aussi observons-nous que les petits de maternelle relèvent surtout la dimension des caractères graphiques et la présence de mots au lieu de lettres: "[dans] les livres pour enfants, les lettres sont écrites plus grosses" et "les livres d'adultes ont comme des mots, pis les enfants ont [...] des lettres". Aux deux cycles suivants, les élèves relèvent surtout des différences dans la numérotation de certaines pages et dans le choix des mots: "[Dans] les livres pour adultes, les numéros sont pas pareils. Des fois, ils peuvent faire des $\mathrm{X}$ à la place des numéros ", et ils ajoutent: "Les mots sont plus longs comme on est gai au lieu de joyeux" et

11. Isabelle Smadja, Harry Potter, les raisons d'un succès, Paris, Presses universitaires de France, coll. "Sociologie d'aujourd'hui ", 2001. 
"dans les livres pour adultes, il y a des mots vulgaires". Ici, malgré la maladresse de l'expression, on comprend que pour ces élèves il est clair que l'écrivain pour enfant vá choisir ses mots en fonction de son jeune lecteur, afin de ne pas le choquer ou lui donner le mauvais exemple.

Quant aux différences liées aux indices structuraux, si certains élèves semblent maîtriser le vocable "structure", nous pouvons difficilement interpréter les connotations qu'ils prêtent à ce terme, surtout quand ils se contentent de dire "La structure est pas pareille". Cependant, certains de leurs commentaires permettent de voir que le nombre de personnages s'avère un critère (sujet à caution) qui distingue les destinataires enfants ou adultes du livre. Certains élèves disent que ales livres pour adultes, i ont pas beaucoup de personnages "; par contre, dautres ajoutent que "[...] dans les livres pour enfants, y en a plusse".

\section{Les supports de récits}

Certains répondants étendent le concept de livre à d'autres supports de récits comme le film, que les parents censurent: "quand y a des monstres [dedans], i disent: "tu peux pas!" "ou encore comme certains sites informatiques pour enfants: "quand on va à l'ordinateur, y a Adibou». Le livre constituerait donc un support d'émotions parmi d'autres pour ces élèves qui le confondraient inconsciemment avec d'autres supports comme le film ou Internet.

\section{Les expériences personnelles}

Finalement, bon nombre d'écoliers ont recours à leur vécu pour répondre à cette question. Les plus jeunes se réfèrent à leur propre habileté en lecture qu'ils expriment en fonction de leur lenteur à déchiffrer les mots: "Un livre pour adultes, c'est plus long à lire, et un livre pour enfants, c'est moins long à lire». Certains élèves plus âgés ont donné le titre d'un livre qu'ils avaient lu: "Il y a un livre que j'ai lu, c'était étonnant, c'était La trilogie de l'aigle noir. Certains autres en indiquent la provenance et commencent à en raconter l'histoire: "Ma mère m'a prêté un livre; c'était [l'histoire d'] une personne qui avait des sens dix fois plus développés [...]”. D'autres, enfin, relèvent la langue dans laquelle l'ouvrage est écrit et son apparence vieillotte comme critères 
paradoxaux: "Moi, j'ai un livre [...], puis c'est en anglais", ou encore formulent la nuance suivante à savoir que, même vieux, les livres peuvent intéresser les enfants: "Y a aussi des livres comme pour adultes qui sont intéressants. Mon père y en a beaucoup des livres de l'ancien temps".

De telles réponses montrent que certains élèves, même très jeunes, semblent capables d'observations nuancées et pertinentes, tandis que d'autres témoignent de leur maladresse à se distancier de l'objet observé. Enfin, à travers leurs réponses à cette quatrième question, certains écoliers manifestent aussi leur fierté d'être lecteurs, et même celle de posséder des livres d'apparence hermétique ou austère.

\section{Conclusion}

D'une manière générale, les filles donnent un peu plus souvent que les garçons leur avis sur la question de l'écrivain et de l'écrit, mais la teneur de leur réponse diffère peu.

Pour ce qui est de l'écrivain, les élèves de cinq à neuf ans se le représentent sous plusieurs aspects. Ils lui reconnaissent a) des objets d'écriture particuliers, b) un âge indéterminé, à la condition qu'il reste sensible à ce qui intéresse les jeunes, c) des sources d'inspiration nombreuses et variées.

Pour ce qui est de l'écrit, si certains semblent capables de distinguer les différents destinataires d'un livre selon des critères objectifs, comme l'iconographie, l'aspect extérieur du livre, voire certains de ses contenus thématiques comme l'amour, la violence et la guerre, d'autres fournissent des réponses plus subjectives en se référant davantage à leur propre vécu. Ils font alors appel à leur propre rythme de lecture, ainsi qu'à leurs intérêts personnels en matière de lecture. Enfin, certains comparent les livres à d'autres supports susceptibles de susciter des émotions, comme le film ou Internet.

Cette analyse des propos des enfants de cinq à neuf ans non seulement nous permet de construire un vaste réseau de leurs représentations de l'écrivain et de l'écrit, mais aussi montre que plus ils sont jeunes, plus leurs représentations se dissocient difficilement de leur environnement immédiat. Il faut attendre l'âge de neuf ans, par exemple, pour obtenir des réponses nuancées comme celles-ci: "La violence, il y en a dans les deux cas. Les 
violences sont de plus haut niveau pour les adultes, tandis que pour les enfants, la violence est plus basse".

Ajoutons que nos résultats montrent que, s'il arrive parfois que les élèves placés en situation d'enseignement réciproque s'influencent les uns les autres dans leurs réponses, ils le font moins par psittacisme que par extension ${ }^{12}$. En effet, plus les élèves vieillissent, plus ils ont tendance à fournir des réponses originales, tout en respectant les limites du champ sémantique donné.

Par ailleurs, les résultats montrent que les élèves sont davantage portés à étendre horizontalement le réseau plutôt qu'à le construire verticalement. En d'autres termes, dans un premier temps, ils semblent plus portés à exprimer des connotations de même niveau conceptuel. Les élèves risqueront une réponse qui relèvera d'un autre niveau de compréhension seulement lorsqu'ils estimeront avoir épuisé le filon. Par exemple, il suffit qu'un élève commence à parler d'un type d'ouvrage susceptible d'être produit par l'écrivain pour que les autres élèves poursuivent l'inventaire cléjà amorcé. Ils ont ainsi tendance à nommer tous les objets d'écriture qu'ils connaissent avant d'aborder d'autres éléments constitutifs du concept étudié. Ainsi, la référence au processus d'écriture n'apparâtit dans leurs énoncés qu'une fois épuisé leur inventaire des objets d'écriture, comme "une plume", "des petites machines", "un crayon à mine" avec lesquels peuvent écrire les écrivains.

Enfin, une telle recherche permet de prendre non seulement la mesure de l'étendue et de la complexité du réseau conceptuel dont disposent les élèves à l'égard de l'écrivain, mais aussi de la compétence des jeunes enfants à se représenter le phénomène plus général de l'écrit. Ce dernier constat illustre sans équivoque que, même si la littérature de jeunesse est encore considérée par beaucoup comme une paralittérature, elle n'en constitue pas moins, à lécole, un objet de savoir qui contribue incontestablement au développement culturel de l'élève. Non seulement elle le familiarise avec la culture en tant qu'objet déjà institutionnalisé ou construit, mais encore elle lui permet d'entrer en relation dynamique avec le monde, avec l'autre et avec sa propre image de soi. Bref, elle participe à son épanouissement.

12. Nicole Girard et Claude Simard, Le feed-back dans la commtication orale, Québec, PPMF |Programme dè perfectionnement des maîtres en français] Lilval, Éditions Ville-Marie, 1981. 